\title{
Estabilidad temporal y validez discriminante del Inventario de Ansiedad de Beck
}

\author{
Temporal stability and discriminant validity of the Beck Anxiety Inventory
}

\author{
Alejandro Emilio Pagano ${ }^{\mathrm{a}, *}$, Nicolás Alejandro Vizioli ${ }^{\mathrm{a}}$ \\ ${ }^{a}$ Facultad de Psicología, Universidad de Buenos Aires, Argentina
}

\begin{abstract}
Resumen
Antecedentes: los trastornos de ansiedad son los de mayor prevalencia a nivel mundial seguidos por los trastornos depresivos. El Inventario de Ansiedad de Beck (BAI; Beck et al., 1988) ha sido elaborado para evaluar la severidad de los síntomas ansiosos y realizar diagnóstico diferencial entre trastornos de ansiedad y depresión. Objetivo: examinar la estabilidad temporal del BAI en su versión adaptada a Argentina y obtener evidencias de validez discriminante. Método: en una muestra de 52 personas se analizó el coeficiente de correlación intraclase. Luego, en una muestra de 225 personas se realizó un análisis de correlaciones entre puntajes del BAI y el Inventario de Depresión de Beck-II (BDI-II; Beck et al., 2006); posteriormente se realizó un análisis factorial exploratorio (AFE) con reactivos de ambos instrumentos y se calculó la proporción Heterotrait-Monotrait Ratio (HTMT). Resultados: el coeficiente de correlación intraclase fue de .82 (IC 95\% = .69 - .90). La correlación entre el BAI y el BDI fue de .56 (tamaño de efecto moderado), el AFE evidenció dos factores bien diferenciados y se halló una proporción HTMT de .66 (IC 95\% = .55 - .74). Conclusiones: se observa una adecuada estabilidad temporal y se aporta evidencia de la validez discriminante del instrumento.
\end{abstract}

Palabras clave: estabilidad temporal; validez discriminante; BAI; ansiedad, depresión.

Para citar este artículo:

Pagano, A. E., \& Vizioli, N. A. (2021). Estabilidad temporal y validez discriminante del Inventario de Ansiedad de Beck. Liberabit, 27(1), e450. https://doi.org/10.24265/liberabit.2021.v27n1.03

\begin{abstract}
Background: Anxiety disorders are the most prevalent worldwide, followed by depressive disorders. The Beck Anxiety Inventory (BAI; Beck et al., 1988) has been developed to assess the severity of anxiety symptoms and perform a differential diagnosis between anxiety and depression disorders. Objective: To examine the temporal stability of the BAI in its version adapted to Argentina and obtain evidence of discriminant validity. Method: In a sample of 52 people, the intraclass correlation coefficient was analyzed. Then, in a sample of 225 people, a correlation analysis between BAI scores and the Beck Depression Inventory-II (BDI-II; Beck et al., 2006) was performed; subsequently, an exploratory factor analysis (EFA) with reagents from both instruments was conducted and the heterotrait-monotrait ratio of correlations (HTMT) was calculated. Results: The intraclass correlation coefficient was .82 (95\% CI = .69 - .90). The correlation between the BAI and the BDI was .56 (moderate effect size). The EFA showed two well-differentiated factors and an HTMT proportion value of .66 (95\% CI = .55 - .74) was found. Conclusions: An adequate temporal stability is observed and evidence of the discriminant validity of the instrument is provided.
\end{abstract}

Keywords: temporal stability; discriminant validity; BAI; anxiety; depression.

Este es un artículo Open Access publicado bajo la licencia Creative Commons Atribución 4.0 Internacional. (CC-BY 4.0)

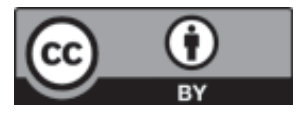

Universidad de San Martín de Porres, Lima - Perú

* paganoalejandro@gmail.com 


\section{Introducción}

El Inventario de Ansiedad de Beck (BAI; Beck et al., 1988) ha sido creado con un doble objetivo: por un lado, proporcionar una medida válida y confiable para medir sintomatología ansiosa; por otro lado, diferenciar la ansiedad de la depresión (Sanz \& Navarro, 2003).

Este segundo objetivo es de gran utilidad práctica debido a la compleja relación existente entre la ansiedad y la depresión. Es preciso subrayar la elevada comorbilidad entre trastornos ansiosos y depresivos (Kessler et al., 2005), ya que gran parte de las personas con trastornos de ansiedad también experimentan trastornos depresivos y viceversa (Gorman, 1996).

Es posible que la elevada comorbilidad entre ambos trastornos se deba a distintos mecanismos en común. Los sesgos cognitivos en el juicio y en la interpretación de las situaciones son comunes para ambos trastornos (Mineka et al., 1998). También lo es el afecto predominantemente negativo.

Ese solapamiento de características ha llevado a la búsqueda de alternativas que permitieran trabajar con la comorbilidad. El surgimiento del enfoque transdiagnóstico de la psicopatología ha posibilitado entender, clasificar e integrar los diferentes síntomas y diagnósticos desde procesos y factores comunes (Sandín et al., 2012). Por esta razón, se han diseñado intervenciones que permitieran trabajar con ambos trastornos, como el protocolo unificado para el tratamiento de trastornos de ansiedad y del estado de ánimo (Barlow et al., 2010).

Otro aspecto a considerar es que se ha reportado que los trastornos de ansiedad son los trastornos de mayor prevalencia a nivel mundial, seguidos por los trastornos depresivos (Ritchie \& Roser, 2018). Situación que se refleja también en la Argentina (Stagnaro et al., 2018). Cabe destacar que, en 2020, a raíz de la pandemia por COVID-19 y los cambios suscitados por los aislamientos sociales (Prieto et al.,
2020), los trastornos cuya prevalencia han aumentado más han sido los de ansiedad y de depresión, según se ha reportado a través de revisiones sistemáticas (Rajkumar, 2020; Salari et al., 2020; Xiong et al., 2020); lo cual constituye una problemática de interés para la salud pública. Dado que los trastornos de ansiedad implican una importante carga individual y social, tienden a ser crónicos y pueden ser incapacitantes (Lépine, 2002). Además, los trastornos de ansiedad están directamente relacionados con la capacidad de trabajo y son de naturaleza crónica, en mayor medida, que los trastornos depresivos (Hendriks et al., 2015). Cabe destacar que los trastornos de ansiedad y de depresión suponen la ejecución de la mayor parte de los recursos económicos destinados al tratamiento de trastornos psicológicos (Ruiz-Rodríguez et al., 2017), aún a pesar de que se ha estimado que solo un cuarto de las personas que cumplen con los criterios de trastornos de ansiedad ha recibido tratamiento (Alonso et al., 2018).

Contar con un instrumento válido y confiable que permita diferenciar ansiedad de depresión constituye un paso importante, tanto para la investigación como para la práctica clínica, al poder orientar mejor las intervenciones. En este sentido, el BAI (Beck et al., 1988) ha ido ganando aceptación profesional, tanto en la formación académica como en la clínica (Piotrowski, 2017); por lo que se han realizado distintas investigaciones acerca de su capacidad de discriminación. Gran parte de estos trabajos se realizaron entre medidas de ansiedad y depresión propuestas por el propio Beck.

Desde sus inicios, el BAI tuvo la finalidad de evaluar la severidad de la sintomatología ansiosa y discriminar ansiedad de depresión. En cuanto a este último punto, los autores reportaron que el BAI discriminó entre grupos con diagnósticos de ansiedad de los grupos de diagnóstico no ansiosos, que incluían depresión mayor o trastorno distímico. Además, hallaron coeficientes de correlación de .48 y .25 con las puntuaciones obtenidas a partir del Inventario de 
Depresión de Beck (BDI; Beck \& Steer, 1993) y la Escala de Depresión de Hamilton (1960, versión revisada), respectivamente.

Estos resultados fueron similares a los reportados por Geissner y Huetteroth (2018), quienes reportaron un coeficiente de correlación de .45 entre las puntuaciones del BAI y del Inventario de Depresión de Beck versión II (BDI-II; Beck et al., 2006).

Steer et al. (1999) administraron el BAI y el BDIII a pacientes diagnosticados con varios tipos de trastornos psiquiátricos, hallando un coeficiente de correlación de .59 entre las puntuaciones del BAI y el BDI-II. A su vez, mediante un análisis de ejes principales, hallaron que uno de los ítems del BDI-II cargó en el factor ansiedad; mientras que dos ítems del BAI cargaron en ambos factores (ansiedad y depresión). Esto destacó que todos los ítems correspondientes al BAI cargaron de manera significativa para el factor ansiedad. Mediante un análisis factorial, hallaron un factor latente de segundo orden que explicaba el 55\% de la varianza e incluía tanto a los reactivos del BAI como del BDI-II. Estos resultados son similares a los reportados por Steer et al. (1995), que hallaron un coeficiente de correlación de .58 entre las puntuaciones obtenidas a través del BAI y el BDI. A través de un análisis de ejes principales encontraron que los 21 ítems del BAI cargaban para el factor denominado ansiedad, junto con un ítem del BDI: preocupación somática. Diecisiete de los reactivos del BDI cargaron en el factor depresión. Las excepciones fueron insomnio, pérdida de apetito, pérdida de peso $\mathrm{y}$ preocupación somática. Asimismo, el ítem del BAI miedo a perder el control cargó, significativamente, en el factor depresión. También hallaron la existencia de un factor de segundo orden que incluía tantos a los ítems del BAI como a los del BDI.

Sanz y Navarro (2003) examinaron las propiedades psicométricas de una versión española del BAI. Hallaron una consistencia interna de alfa = .88 y una solución factorial de una única dimensión de ansiedad compuesta por dos factores interrelacionados correspondientes a síntomas somáticos y afectivo-cognitivos. Tomando el DSMIV (American Psychological Association, 1994), encontraron que la validez de contenido del BAI fue adecuada, ya que sus ítems cubrían el 45\% de los criterios sintomáticos específicos de los trastornos de ansiedad y el 78\% de los síntomas de los ataques de pánico. En cuanto a la validez discriminante del BAI, hallaron un coeficiente de correlación de .58 con la puntuación obtenida a partir de la administración del BDI-II. Asimismo, a través de un análisis factorial combinado de los ítems del BAI y del BAI-II, hallaron la existencia de dos factores diferenciados uno de ansiedad y uno de depresión.

A su vez, Sanz et al. (2012), hallaron un coeficiente de correlación de .63 entre las puntuaciones del BAI y del BDI-II. A través de un análisis factorial que incluyó a los reactivos del BAI y del BDI-II, hallaron que todos los reactivos correspondientes al BAI cargaron en un solo factor de manera significativa, con excepción de rubor facial, cuya carga factorial no fue satisfactoria para ninguno de los dos factores. En cuanto al BDI-II, los ítems cargaron en un segundo factor de manera significativa, con excepción del ítem agitación, cuya carga fue significativa para el primer factor. El ítem cambios en el apetito no cargó de manera satisfactoria para ninguno de los dos factores.

Por otro lado, Lee et al. (2016) hallaron un coeficiente de correlación de .58 entre las puntuaciones del BAI y la medida de depresión Cuestionario de Salud del Paciente-9 (PHQ-9; Kroenke et al., 2001).

Guillén y González-Celis (2019) hallaron un coeficiente de correlación entre las puntuaciones del BAI y del BDI-II de .53. Por su parte, en un metaanálisis psicométrico de versiones en lengua inglesa del BAI, Bardhoshi et al. (2016) reportaron un coeficiente de correlación agregado de .59 entre puntuaciones obtenidas a través del BAI y el BDI-II. 
Como puede observarse, las asociaciones resultantes entre las puntuaciones obtenidas entre el BAI y distintas medidas de depresión tienden a ser moderadas. Lo mencionado podría interpretarse como indicación de problemas en la validez discriminante o como evidencia acerca de relación existente entre la ansiedad y la depresión (Lee et al., 2016). Para intentar determinar esta cuestión, distintas investigaciones han recurrido a la realización de análisis factoriales que incluyeran tanto a los reactivos del BAI y del BDI-II, a fin de determinar la capacidad discriminativa al nivel de los ítems. Basándose en que la validez discriminante puede calcularse tanto al nivel de los constructos como al nivel de los ítems (Voorhees et al., 2016), se considera esperable la existencia de asociaciones entre la ansiedad y la depresión (Sanz et al., 2012) y que aun así los reactivos de los instrumentos que las evalúan tengan capacidad discriminativa. Sin embargo, no se han encontrado investigaciones que incluyan al método para estudiar la validez discriminante que se recomienda actualmente: el cálculo de la proporción heterorasgo-monorasgo (HTMT, Heterotrait-Monotrait Ratio), propuesta por Henseler et al. (2015).

Otra de las propiedades psicométricas que debe tomarse en cuenta para determinar la confiabilidad de un instrumento es su estabilidad temporal. En cuanto a las evidencias de estabilidad temporal del BAI, Beck et al. (1988) hallaron un coeficiente de correlación de .75 en una muestra de 83 pacientes psiquiátricos que completaron el BAI dos veces con una diferencia de una semana. A través de un metaanálisis psicométrico, Bardhoshi et al. (2016) reportaron una estabilidad temporal agregada de .65, sintetizando 18 estudios que la indagaban, con una media y mediana de espacio temporal entre aplicaciones del instrumento de seis semanas. Sin embargo, son pocas las investigaciones que indagaron la estabilidad temporal del BAI. Por esta razón, los objetivos de la presente investigación son a) examinar la estabilidad temporal de las puntuaciones del BAI y b) obtener evidencias de acerca de su validez discriminante.

\section{Método}

\section{Participantes}

Para analizar las evidencias de validez discriminante, se trabajó con un muestreo intencional no probabilístico, mediante el cual se recogió una muestra de 225 personas cuyas edades estaban comprendidas entre los 18 y 63 años $(M=33.02 ; D E$ = 11.89), de los cuales el 58\% eran mujeres y el $42 \%$ varones. El 53\% residía en la Ciudad de Buenos Aires y el $47 \%$ en el Conurbano Bonaerense. El 43\% trabajaba en relación de dependencia en calidad de empleado, el 35\% trabajaba en calidad de independiente, el $16 \%$ era estudiante, el $4 \%$ estaba desocupado y el $2 \%$ era jubilado. En relación al nivel de instrucción, el 37\% poseía estudios universitarios incompletos; el 26\%, estudios universitarios; el 9\%, posgrado; el 9\%, terciario incompleto; el 9\%, secundario completo; el $8 \%$, terciario completo; y el $2 \%$, secundario incompleto. Asimismo, el 52\% manifestó estar soltero; mientras que el $27 \%$, en pareja; el $17 \%$, casado; el $3 \%$, divorciado; y el $1 \%$ restante se dividió entre viudos y separados.

Para analizar la estabilidad temporal de las puntuaciones del BAI, se trabajó con una muestra de 52 participantes cuyas edades iban desde los 21 a los 63 años $(M=30.46 ; D E=10.65)$, de los cuales el $83 \%$ eran mujeres y el $17 \%$ eran varones. El $60 \%$ residía en la Ciudad de Buenos Aires y el $40 \%$ en el Conurbano Bonaerense. Respecto de la ocupación, el $40 \%$ refirió trabajar en relación de dependencia en calidad de empleado, el $29 \%$ ser estudiante, el 23\% ser independiente, el $6 \%$ estar jubilado y el $2 \%$ encontrarse desocupado. En relación al nivel de instrucción, el 54\% refirió poseer estudios universitarios incompletos; el 25\%, estudios universitarios completos; el $13 \%$, posgrado; el $6 \%$, terciario; y el $2 \%$, secundario completo. El 54\% de los participantes refirió estar soltero, el 32\% encontrarse en pareja, el $8 \%$ estar casado y el $6 \%$ haberse divorciado. 


\section{Instrumentos}

Inventario de Ansiedad de Beck (BAI; Beck et al., 1988; adaptación argentina: Vizioli \& Pagano, 2020). Es un instrumento autoadministrable de 21 ítems que miden síntomas característicos de la ansiedad. La puntuación de los reactivos se realiza sobre una escala Likert de 4 opciones (de 0 para nada a 3 severamente - me molestó mucho). El inventario presenta adecuadas propiedades psicométricas para la población argentina, con evidencias de validez de constructo a través de análisis factoriales confirmatorios, y una consistencia interna de alfa ordinal $=.93 \mathrm{y}$ un omega ordinal $=.95$.

Inventario de Depresión de Beck (BDI-II; Beck et al, 2006; adaptación argentina: Brenlla \& Rodríguez, 2006). Se trata de un inventario autoadministrable que consta de 21 ítems que hacen referencia a síntomas característicos de la depresión. La puntuación de los reactivos se realiza sobre una escala Likert de 4 opciones (0 a 3). La puntuación total varía de 0 a 63, teniendo en cuenta puntos de corte según la gravedad e intensidad de síntomas depresivos: 0 - 13 = depresión mínima, 14 - 19 = depresión leve, 20 - 28 = depresión moderada y 29 - 63 = depresión severa. El inventario presenta adecuadas propiedades psicométricas para la población argentina, con una consistencia interna de alfa de Cronbach $=.88$.

\section{Procedimiento}

La recolección de datos fue responsabilidad de los autores del presente manuscrito y se realizó mediante plataforma virtuales. Se realizaron dos administraciones con una diferencia de tres meses. En ambos casos se incluyó un consentimiento informado en el cual se especificaron los detalles de los objetivos y los lineamientos generales de la investigación, junto con las garantías de confidencialidad. Se explicó a los participantes que podían abandonar la participación en el momento en que lo consideren.

Con respecto al consentimiento informado, se explicitó las características de la participación, que fue anónima, voluntaria y sin compensación. Los participantes dieron su consentimiento mediante la selección de una de dos opciones de respuesta al comienzo de la administración: Deseo participar y No deseo participar. La selección de la primera respuesta permitía completar los instrumentos, mientras que la elección de la segunda finalizaba la administración.

\section{Análisis de datos}

Para analizar la estabilidad temporal se calculó el coeficiente de correlación intraclase de las administraciones del BAI, siguiendo el procedimiento recomendado para analizar la confiabilidad test-retest (Polit, 2014). De acuerdo a las indicaciones de Trevethan (2017), se reportaron las medidas promedio con un intervalo de confianza de 95\% del modelo mixto de dos vías (two way). Se consideraron aceptables valores mayores a .70 (De Vet et al., 2011) y se interpretaron los coeficientes siguiendo los lineamientos sugeridos por Koo y Li (2016), quienes especificaron que los valores inferiores a .50 indican una confiabilidad deficiente; los valores entre .50 y .75, una confiabilidad moderada; los valores entre .75 y .90 , una buena confiabilidad; y los valores superiores a .90, una confiabilidad excelente.

El análisis de validez discriminante se realizó, en primer lugar, a través del análisis de correlaciones de Pearson entre los puntajes obtenidos en el BAI y el BDI-II. Para los análisis de correlaciones se tomó en cuenta un nivel de significación .05. Para estimar el tamaño del efecto de las correlaciones se utilizó el criterio propuesto por Dancey y Reidy (2007), considerando tamaños de efecto fuertes a $r>.70$, tamaños de efectos moderados a $r>.40$ y tamaños de efecto débiles a $r>.10$; no obstante, debe ser considerado que los puntos de corte no son absolutos y dependen de la disciplina en la cual se esté trabajando (Akoglu, 2018). Todos los cálculos de correlaciones se realizaron mediante el software SPSS versión 26.

En segundo lugar, se procedió a analizar la validez discriminante de los reactivos mediante un análisis 
factorial exploratorio (AFE), incluyendo a los ítems correspondientes tanto del BAI como del BDI-II. Si bien se hicieron modificaciones en el AFE, se siguió el procedimiento propuesto por Sanz et al. (2012). Para determinar el número de dimensiones a extraer, se utilizó el test de mínima correlación parcial promediada de Velicer (MAP) uno de los procedimientos indicados para evaluar la dimensionalidad de una matriz de datos (Zwick \& Velicer, 1986). Se trabajó sobre una matriz de correlaciones policóricas (Ferrando \& Lorenzo-Seva, 2014) con el programa Factor versión 10 (LorenzoSeva \& Ferrando, 2020). Con la solución factorial indicada de acuerdo al MAP, se realizó un AFE con método de extracción de mínimos cuadrados no ponderados, siguiendo las recomendaciones de LloretSegura et al. (2014). Para conseguir mayor simplicidad e interpretabilidad, se optó por trabajar con la solución rotada mediante rotación oblicua promax, de acuerdo al criterio sugerido por Lloret-Segura et al. (2017). Se consideraron aceptables cargas factoriales mayores a .32 (Tabachnick \& Fidell, 2001). A su vez, se examinó la validez teórica de los ítems en relación a su carga factorial, privilegiando la interpretación teórica de los resultados obtenidos (Lloret-Segura et al., 2014).

Adicionalmente, en tercer lugar, se calculó la proporción HTMT. Esta es la media de las correlaciones existentes entre indicadores que miden diferentes constructos, en relación con el promedio de las correlaciones de indicadores dentro del mismo constructo. El cálculo de la proporción HTMT se ha indicado por encima de otros métodos que presentan dificultades en diversas circunstancias en términos de sensibilidad y especificidad (Hair et al., 2017a; Hair et al., 2017b). Inclusive, su superioridad se ha demostrado en base a resultados obtenidos a partir de simulaciones (Voorhees et al., 2016). Por estas razones, se optó por el cálculo de la proporción HTMT para obtener evidencias de la validez discriminante del BAI.

Henseler et al. (2015), informaron que valores inferiores a .90 evidencian una adecuada validez discriminante. Por su parte, Kline (2011) recomienda que los valores obtenidos no superen el límite de .85, para asegurar la ausencia de problemas en la capacidad de discriminación. Mediante el método de bootstrapping (muestra = 4999 veces), se realizó el cálculo de los intervalos de confianza no paramétricos de la proporción HTMT, cuyos valores deberían ser en todos los casos menores a 1 (Benitez et al., 2020). Los cálculos se realizaron mediante el software ADANCO (Henseler, 2017).

\section{Resultados}

Con respecto a la estabilidad temporal del BAI, la Tabla 1 refleja que el coeficiente de correlación intraclase ha sido aceptable, indicando una buena confiabilidad.

Tabla 1

Análisis de correlaciones intraclase de las puntuaciones obtenidas con el BAI

\begin{tabular}{lcccccc}
\hline & CICC & IC 95\% (min - máx) & F & gl1 & gl2 & Sig \\
\hline Puntuación BAI & .82 & $(.69-.90)$ & 5.66 & 51 & 51 & $<.001$
\end{tabular}

Nota: CICC = coeficiente de correlación intraclase; IC = intervalo de confianza; min = límite inferior; máx = límite superior; gl = grados de libertad; sig = nivel de significación. Análisis realizado sobre la muestra $n=52$.

Respecto de la validez discriminante del BAI, se obtuvo un coeficiente de correlación de Pearson de $.56(p<.05)$ entre las puntuaciones del BAI y del BDIII, con un tamaño de efecto moderado $(n=225)$. 
A nivel de reactivos, la validez discriminante se estudió mediante un AFE que incluyó a los ítems del BAI y del BDI-II. Antes de comenzar el AFE, se estudió la adecuación de los datos para el trabajo con matrices policóricas, prueba de esfericidad de Bartlett y la medida de adecuación de Kaiser-Meyer-Olkin; y se examinó la dimensionalidad mediante el test de MAP. En la prueba de esfericidad de Bartlett se obtuvo un chicuadrado de $2419.6(p<.05)$, que implica que la matriz de correlación se diferencia significativamente de la matriz unidad. Respecto de la medida de adecuación de Kaiser-Meyer-Olkin, se obtuvo un valor KMO = .87, que indica la adecuación general de la matriz. En cuanto al test de MAP, se evidenció la adecuación de una solución de dos factores.

La Tabla 2 muestra las cargas factoriales de los ítems del BAI y del BDI-II. Como puede observarse, los ítems del BAI tuvieron valores considerados aceptables en todos los casos, contribuyendo al mismo factor (ansiedad). En caso de los ítems correspondientes al BDI-II, el ítem agitación cargó para el factor ansiedad. El ítem cambios en los hábitos de sueño no exhibió cargas aceptables para ninguno de los dos factores. Los demás ítems del BDI-II exhibieron cargas factoriales aceptables para el factor depresión. Es decir que los ítems del BAI exhiben una adecuada capacidad discriminante en relación a la sintomatología depresiva.

La proporción HTMT de la correlación entre las puntuaciones del BAI y del BDI-II fue de .66, con intervalos de confianza $=.55-.74$, es decir, por debajo de los límites sugeridos. Esto sugiere la existencia de evidencias de validez discriminante.

Tabla 2

Cargas factoriales del AFE realizado con los ítems del BAI y del BDI II

\begin{tabular}{lll}
\hline \multicolumn{1}{c}{ Ítem } & Factor 1 (ansiedad) & Factor 2 (depresión) \\
\hline 1. Hormigueo o entumecimiento & .37 \\
2. Sensación de calor intenso & .42 \\
3. Debilidad en las piernas & .36 \\
4. Incapacidad para relajarse & .52 \\
5. Miedo a que pase lo peor & .61 \\
6. Mareos o vértigos & .63 \\
7. Palpitaciones o taquicardia & .62 \\
8. Sensación de inestabilidad & .56 \\
9. Con miedo o aterrorizado/a & .60 \\
10. Nervioso/a & .53 \\
11. Sensación de ahogo & .52 \\
12. Temblor de manos & .63 \\
13. Inquieto/a, tembloroso/a & .56 \\
14. Miedo a perder el control & .69 \\
15. Dificultad para respirar & .63 \\
16. Miedo a morir & .62 \\
17. Asustado/a & .61 \\
18. Indigestión o malestar estomacal & .48 \\
19. Con desvanecimientos o desmayos & .56 \\
20. Ruborizarse, sonrojamiento & .48 \\
21. Sudoración (no producida por calor) & .54
\end{tabular}




\begin{tabular}{|c|c|c|}
\hline Ítem & Factor 1 (ansiedad) & Factor 2 (depresión) \\
\hline 1. Tristeza & & .57 \\
\hline 2. Pesimismo & & .66 \\
\hline 3. Fracaso & & .51 \\
\hline 4. Pérdida de placer & & .61 \\
\hline 5. Sentimientos de culpa & & .43 \\
\hline 6. Sentimientos de castigo & & .45 \\
\hline 7. Disconformidad con uno mismo & & .80 \\
\hline 8. Autocrítica & & .48 \\
\hline 9. Pensamiento o deseos suicidas & & .39 \\
\hline 10. Llanto & & .41 \\
\hline 11. Agitación & .41 & \\
\hline 12. Pérdida de interés & & .56 \\
\hline 13. Indecisión & & .58 \\
\hline 14. Desvalorización & & .70 \\
\hline 15. Pérdida de energía & & .55 \\
\hline \multicolumn{3}{|l|}{ 16. Cambios en los hábitos de sueño } \\
\hline 17. Irritabilidad & & .39 \\
\hline 18. Cambios de apetito & & .38 \\
\hline 19. Dificultad de concentración & & .64 \\
\hline 20. Cansancio o fatiga & & .42 \\
\hline 21. Pérdida de interés en el sexo & & .34 \\
\hline
\end{tabular}

Nota: AFE realizado sobre la muestra $n=225$

\section{Discusión}

La presente investigación se propuso examinar la estabilidad temporal de las puntuaciones del BAI, así como obtener evidencias de validez discriminante. En cuanto al primer objetivo, se han obtenido evidencias de una buena estabilidad temporal. Esto implica que las fuentes de error que se experimentan con los cambios temporales no afectaron significativamente a las puntuaciones (Irwin \& Hughes, 2018). Estos resultados son superiores a los reportados por Beck et al. (1988), así como a los hallados por Bardhoshi et al. (2016) a través de su metaanálisis psicométrico, aportando evidencias acerca de la estabilidad temporal del BAI.

Con respecto a la validez discriminante del BAI, en primer lugar, se obtuvo una correlación significativa con un tamaño de efecto moderado con las puntuaciones del BDI-II. El coeficiente de correlación en cuestión fue de .56. Se puede observar en la literatura diferentes estudios donde esta correlación fue levemente superior. En los trabajos realizados por Steer et al. (1999) con 840 pacientes ambulatorios y Steer et al. (1995) con 1000 pacientes ambulatorios, en ambos casos diagnosticados con varios tipos de trastornos psiquiátricos, las correlaciones reportadas fueron de .59 y .58, respectivamente. También el estudio realizado por Sanz et al. (2012) con 307 pacientes españoles que padecían trastornos psicológicos demostró una correlación de .63. A su vez, existen estudios realizados con población general que también ha demostrado una correlación superior. Tal es el caso de la investigación realizada por Sanz y Navarro (2003) con 590 estudiantes universitarios 
españoles y Lee et al. (2016) con 1022 residentes de Seúl Incheon, Cheongju, Daegu y Jinju, en Corea, con coeficientes de .58 hallados en ambos estudios.

Sin embargo, la correlación encontrada en la presente investigación es sensiblemente mayor al coeficiente de .53 hallado por Guillén y González-Celis (2019) en una muestra con 157 usuarios asmáticos de un instituto de salud pública en México. Mientras que también supera al coeficiente de .45 comunicado por Geissner y Huetteroth (2018) en un estudio realizado con tres muestras que en total incluyeron 409 pacientes diagnosticados con trastornos de ansiedad. Incluso, se observa que el coeficiente de correlación supera a los reportados por Beck et al. (1988) de .25 y .48 en la validación del inventario original.

El resultado obtenido a partir del análisis de correlaciones entre ambos constructos podría interpretarse de dos formas: como validez discriminante insuficiente o como un reflejo de la relación existente entre ansiedad y depresión (Lee et al., 2016). Para responder a esta cuestión, se realizó un análisis de la capacidad discriminativa de los ítems a partir de una AFE y se calculó la proporción HTMT.

En cuanto al AFE, mostró que los ítems del BAI se agrupaban en conjunto en un mismo factor, la ansiedad, con cargas factoriales aceptables en todos los casos; aportando evidencias de la capacidad discriminativa de los reactivos que componen al instrumento. Lo que puede sugerir que las asociaciones existentes entre los constructos subyacentes pueden deberse en mayor medida a la naturaleza de su relación, que a la capacidad de discriminación del BAI. Cabe destacar que hubo dos ítems del BDI-II cuyas cargas no fueron las esperables: agitación y cambios en los hábitos del sueño. El primero evidenció una carga factorial satisfactoria para el factor ansiedad, mientras que el segundo no ofreció una carga factorial satisfactoria para ninguno de los dos factores. Los resultados obtenidos con el ítem agitación son similares a los reportados por Steer et al. (1999) y Sanz et al. (2012).
La investigación de Steer et al. (1999) se realizó con 840 pacientes ambulatorios que fueron diagnosticados con varios tipos de trastornos psiquiátricos, con la finalidad de determinar la composición general de un factor de malestar general y las dimensiones específicas de depresión y ansiedad. Mediante un análisis de ejes principales que incluyó a los ítems del BAI y del BDI-II, reportaron que 20 de los ítems del BDI-II se cargaron en el factor depresión, con la excepción del reactivo agitación.

Para interpretar este resultado, es preciso recordar que este reactivo incluye las siguientes opciones de respuesta: no estoy más inquieto o tenso que lo habitual, me siento más inquieto o tenso que lo habitual, estoy tan inquieto o agitado que me es difícil quedarme quieto y estoy tan inquieto o agitado que tengo que estar siempre en movimiento o haciendo algo. De manera tal que pueden estar solapándose con características de la ansiedad medidas por ítems del BAI, como pudieran ser inquieto/a, tembloroso/a o incapacidad para relajarse. En este sentido, también debe destacarse que el DSM 5 (American Psychiatric Association, 2013) incluye a la inquietud o impaciencia como uno de los síntomas característicos del Trastorno de Ansiedad Generalizada. Ello podría explicar que ese reactivo cargue satisfactoriamente para el factor ansiedad. En cuanto a la carga factorial no aceptable para ninguno de los dos factores correspondiente al ítem cambios en los hábitos del sueño, estos resultados coinciden parcialmente con los hallados por Steer et al. (1995), donde el reactivo insomnio del BDI fue uno de los que no cargó para el factor depresión.

Con respecto a la proporción HTMT, el valor obtenido fue menor a los límites sugeridos en la literatura investigativa. Tanto para el valor de la propia proporción $(<.85)$ como para los intervalos de confianza obtenidos $(<1)$. Lo cual implica que cada constructo latente comparte más varianza con sus propios indicadores o reactivos, que con los otros 
(Henseler et al., 2015). De manera que la proporción HTMT mostró evidencias acerca de la adecuada validez discriminante del BAI. Si bien no se habían encontrado investigaciones que utilizaran este método, los resultados aportan nueva información que sostiene la utilidad del BAI para hacer frente a los objetivos que impulsaron su construcción: medir la severidad de la ansiedad y proporcionar una medida que permita discriminar a la ansiedad de la depresión (Beck et al., 1988).

La presente investigación ha aportado evidencias acerca de la buena estabilidad temporal de la versión argentina del BAI, así como de su adecuada capacidad de discriminación. Hallazgos que aportan nueva información acerca de su validez y confiabilidad. El BAI puede ser un instrumento muy útil en el ámbito de la salud pública debido a la facilidad de su administración y a su brevedad. En particular, la validez discriminante es de gran importancia para distinguir ansiedad de depresión y poder orientar mejor el trabajo psicoterapéutico.

En cuanto a las limitaciones del presente estudio, en primer lugar, se debe manifestar las dificultades halladas al intentar brindar evidencias de la capacidad de discriminación del BAI respecto de la sintomatología depresiva. Las mismas son evidenciadas por el hallazgo de un coeficiente de correlación moderado entre el BAI y el BDI-II, que se asemeja a los encontrados en la literatura. No obstante, al nivel de los ítems, el AFE que incluyó a los reactivos del BAI y del BDI arrojó dos factores bien diferenciados, con cargas satisfactorias en todos los reactivos del BAI. A su vez, el cálculo de la proporción HTMT permitió obtener nueva evidencia sobre la validez discriminante del BAI, con la importancia de tratarse de un método recomendado por encima de otros por su sensibilidad y especificidad (Hair et al., 2017a; Hair et al., 2017b). En conclusión, los hallazgos pueden indicar que el coeficiente de correlación moderado está ligado a la relación existente entre ansiedad y depresión más que a la capacidad de discriminación del BAI.
En segundo lugar, pueden señalarse el reducido tamaño muestral y la no representatividad de la muestra. Por lo que futuras investigaciones podrían incluir la utilización de un método probabilístico en la recolección de datos y asegurar la representatividad de la muestra.

Por último, podrían incluirse más intervalos temporales para estudiar con mayor precisión la estabilidad temporal del BAI y ganar especificidad acerca del impacto de los efectos del error asociados al paso del tiempo. Finalmente, se sugiere que futuras investigaciones examinen la validez convergente e incremental del BAI.

\section{Conflicto de intereses}

Los autores no reportan conflictos de intereses.

\section{Responsabilidad ética}

El presente trabajo se realizó considerando la Declaración de Helsinki (World Medical Association, 2013), que establece los principios éticos fundamentales para la investigación con seres humanos. No se realizaron experimentos que dañasen o pusieran en riesgo a personas, todas leyeron y llenaron un consentimiento informado.

\section{Confidencialidad de los datos}

Los datos fueron recolectados mediante plataformas virtuales. Se incluyó en los formularios la garantía de confidencialidad, no se solicitaron datos que pudieran reconocer la identidad de los participantes de dicho estudio. Para el estudio que implicó un retest se solicitó a los participantes que quisieran volver a participar en una segunda investigación que colocaran una dirección de mail de contacto.

\section{Derecho a la privacidad y consentimiento informado}

Se incluyó un consentimiento informado en el cual se especificaron los detalles de los objetivos y los lineamientos generales de la investigación. Se explicó 
a los participantes que podían abandonar la participación en el momento en que lo consideren. El consentimiento informado explicitó las características de la participación, que fue voluntaria y sin compensación. Los participantes dieron su consentimiento mediante la selección de una de dos opciones de respuesta al comienzo de la administración: Deseo participar y No deseo participar. La selección de la primera respuesta permitía completar los instrumentos, mientras que la elección de la segunda finalizaba la administración. Declaramos que no aparecen datos de los participantes o algún indicio de identificación.

\section{Contribución de la autoría}

AEP: concepción y diseño del estudio, interpretación de los datos y revisión final del manuscrito.

NAV: concepción y diseño del estudio, interpretación de los datos y revisión final del manuscrito.

\section{Referencias}

Akoglu, H. (2018). User's Guide to Correlation Coefficients. Turkish Journal of Emergency Medicine, 18(3), 91-93. https://doi.org/10.1016/j.tjem.2018.08.001

Alonso, J., Liu, Z., Evans-Lack, S., Sadikova, E., Sampson, N., Chatterji, S., Abdulmalik, J., Aguilar-Gaxiola, S., AlHamzawi, A., Andrade, L., Bruffaerts, R., Cardoso, G., Cia, A., Florescu, S., de Girolamo, G., Gureje, O., Haro, J., He, Y., de Jonge, P., ... WHO World Mental Health Survey Collaborators. (2018). Treatment Gap for Anxiety Disorders is Global: Results of the World Mental Health Surveys in 21 Countries. Depression and Anxiety, 35(3), 195-208. https://doi.org/10.1002/da.22711

American Psychiatric Association. (2013). Diagnostic and Statistical Manual of Mental Disorders (DSM-5). American Psychiatric Association.

American Psychological Association. (1994). Diagnostic and Statistical Manual of Mental Disorders (4. ${ }^{\text {a ed.). }}$ American Psychological Association.
Bardhoshi, G., Duncan, K., \& Erford, B. T. (2016). Psychometric Meta-Analysis of the English Version of the Beck Anxiety Inventory. Journal of Counseling \& Development, 94(3), 356-373. https://doi.org/10.1002/ jcad.12090

Barlow, D. H., Ellard, K. K., \& Fairholme, C. P. (2010). Unified Protocol for Transdiagnostic Treatment of Emotional Disorders: Workbook. Oxford University Press.

Beck, A. T., Epstein, N., Brown, G., \& Steer, R. A. (1988). An Inventory for Measuring Clinical Anxiety: Psychometric Properties. Journal of Consulting and Clinical Psychology, 56(6), 893-897. https://doi.org/ 10.1037/0022-006X.56.6.893

Beck, A. T., \& Steer, R. A. (1993). Manual for the Revised Beck Depression Inventory. Psychological Corporation.

Beck, A. T., Steer, R. A., \& Brown, G. K. (2006). Inventario de Depresión de Beck (BDI-II), Manual. Paidós.

Benitez, J., Henseler, J., Castillo, A., \& Schuberth, F. (2020). How to Perform and Report an Impactful Analysis Using Partial Least Squares: Guidelines for Confirmatory and Explanatory IS Research. Information \& Management, 57(2), 103168. https:// doi.org/10.1016/j.im.2019.05.003

Brenlla, M. E., \& Rodríguez, C. M. (2006). Adaptación Argentina del Inventario de Depresión de Beck (BDIII). Paidós.

Dancey, C. P., \& Reidy, J. (2007). Statistics Without Maths for Psychology. Pearson Education.

De Vet, H. C. W., Terwee, C., Mokkink, L. B., \& Knol, D. L. (2011). Measurement in Medicine: A Practical Guide. Cambridge University Press.

Ferrando, P. J., \& Lorenzo-Seva, U. (2014). El análisis factorial exploratorio de los ítems: algunas consideraciones adicionales. Anales de Psicología, 30(3), 1170-1175. https://revistas.um.es/analesps/ article/view/analesps.30.3.199991/165451

Geissner, E., \& Huetteroth, A. (2018). Beck Anxiety Inventory Deutsch - Ein Reliables, Valides und Praxisgeeignetes Instrument zur Messung Klinischer Angst. Psychotherapie, Psychosomatik, Medizinische Psychologie, 68(3-4), 118-125. https://doi.org/10.1055/ s-0043-122941 
Gorman, J. M. (1996). Comorbid Depression and Anxiety Spectrum Disorders. Depression and Anxiety, 4(4), 160168. https://doi.org/10.1002/(SICI)1520-6394(1996)4:4\% 3C160::AID-DA2\%3E3.0.CO;2-J

Guillén, C., \& González-Celis, A. L. (2019). Propiedades psicométricas del Inventario de Ansiedad de Beck en adultos asmáticos mexicanos. Psicología y Salud, 29(1), 5-16. https://psicologiaysalud.uv.mx/index.php/ psicysalud/article/view/2563/4471

Hair, J. F., Hult, G. T. M., Ringle, C. M., \& Sarstedt, M. (2017a). A Primer on Partial Least Squares Structural Equation Modeling (PLSSEM) (2. ${ }^{\mathrm{a}}$ ed.). SAGE Publications, Inc.

Hair, J. F., Matthews, L. M., Matthews, R. L., \& Sarstedt, M. (2017b). PLS-SEM or CB-SEM: Updated Guidelines on which Method to Use. International Journal of Multivariate Data Analysis, 1(2), 107-123. https:/doi. org/10.1504/IJMDA.2017.087624

Hamilton, M. (1960). A Rating Scale for Depression. Journal of Neurology, Neurosurgery, and Psychiatry, 23(1), 56-62. http://dx.doi.org/10.1136/jnnp.23.1.56

Hendriks, S. M., Spijker, J., Licht, C. M., Hardeveld, F., de Graaf, R., Batelaan, N. M., Penninx, B. W., \& Beekman, A. T. (2015). Long-Term Work Disability and Absenteeism in Anxiety and Depressive Disorders. Journal of Affective Disorders, 178, 121-130. https:// doi.org/10.1016/j.jad.2015.03.004

Henseler, J. (2017). Adanco 2.0. 1-User Manual. Composite Modeling GmbH \& Co.

Henseler, J., Ringle, C. M., \& Sarstedt, M. (2015). A New Criterion for Assessing Discriminant Validity in Variance-Based Structural Equation Modelling. Journal of the Academy of Marketing Science, 43(1), 115-135. https://doi.org/10.1007/s11747-014-0403-8

Irwing, P., \& Hughes, D. J. (2018). Test Development. In P. Irwing, T. Booth, \& D. J. Hughes (eds.), The Wiley Handbook of Psychometric Testing (vol. 1, pp. 3-47). Wiley.

Kessler, R. C., Chiu, W. T., Demler, O., \& Walters, E. E. (2005). Prevalence, severity, and comorbidity of 12month DSM-IV disorders in the National Comorbidity Survey Replication. Archives of general psychiatry, 62(6), 617-627.
Kline, R. B. (2011). Principles and Practice of Structural Equation Modeling (3. ${ }^{\text {a }}$ ed.). The Guilford Press.

Koo, T. K., \& Li, M. Y. (2016). A Guideline of Selecting and Reporting Intraclass Correlation Coefficients for Reliability Research. Journal of Chiropractic Medicine, 15(2), 155-163. https://dx.doi.org/10.1016\% 2Fj.jcm.2016.02.012

Kroenke, K., Spitzer, R. L., \& Williams, J. B. (2001). The PHQ-9: Validity of a Brief Depression Severity Measure. Journal of General Internal Medicine, 16, 606-613. https://doi.org/10.1046/j.1525-1497.2001.016009606.x

Lee, H. K., Lee, E. H., Hwang, S. T., Hong, S. H., \& Kim, J. H. (2016). Psychometric Properties of the Beck Anxiety Inventory in the Community-Dwelling Sample of Korean Adults. Korean Journal of Clinical Psychology, 35(4), 822-830. http://kjp-clinical.org/xml/08897/08897.pdf

Lépine, J. P. (2002). The Epidemiology of Anxiety Disorders: Prevalence and Societal Costs. The Journal of Clinical Psychiatry, 14, 4-8.

Lloret-Segura, S., Ferreres-Traver, A., Hernández-Baeza, A., \& Tomás-Marco, I. (2014). El análisis factorial exploratorio de los ítems: una guía práctica, revisada y actualizada. Anales de Psicología, 30(3), 1151-1169. https://revistas.um.es/analesps/article/view/199361

Lloret-Segura, S., Ferreres-Traver, A., Hernández-Baeza, A., \& Tomás-Marco, I. (2017). El análisis factorial exploratorio de ítems: análisis guiado basado en datos empíricos y software. Anales de Psicología, 33(2), 417432. https://revistas.um.es/analesps/article/download/ analesps.33.2.270211/210801/0

Lorenzo-Seva, U., \& Ferrando, P. (2020). Manual of the Program FACTOR v. 10. http://psico.fcep.urv.es/utilitats/ factor/index.html

Mineka, S., Watson, D., \& Clark, L. A. (1998). Comorbidity of Anxiety and Unipolar Mood Disorders. Annual Review of Psychology, 49(1), 377-412. https://doi.org/ 10.1146/annurev.psych.49.1.377

Piotrowski, C. (2017). The Status of the Beck Inventories (BDI, BAI) in Psychology Training and Practice: A Major Shift in Clinical Acceptance. Journal of Applied Biobehavioral Research, 23(3), e12112. https://doi.org/ 10.1111/jabr.12112 
Polit, D. F. (2014). Getting Serious About Test-Retest Reliability: A Critique of Retest Research and Some Recommendations. Quality of Life Research, 23(6), 1713-1720. https://doi.org/10.1007/s11136-014-0632-9

Prieto, D. E., Aguirre, G. L., de Pierola, I., Luna, G., Merea, L., Lazarte, C. S., Uribe-Bravo, K. A., \& Zegarra, Á. C. (2020). Depresión y ansiedad durante el aislamiento obligatorio por el COVID-19 en Lima Metropolitana. Liberabit, 26(2), e425-e425. https://doi.org/10.24265/ liberabit.2020.v26n2.09

Rajkumar, R. P. (2020). COVID-19 and Mental Health: A Review of the Existing Literature. Asian Journal of Psychiatry, 52, 102066. https://doi.org/10.1016/j.ajp.20 20.102066

Ritchie, H., \& Roser, M. (2018). Mental Health. Our World in Data. https://ourworldindata.org/mental-healthj

Ruiz-Rodríguez, P., Cano-Vindel, A., Muñoz, R., Medrano, L., Moriana, J. A., Buiza, C., Jiménez, G., Gonzáles Blanch, C., \& Grupo de Investigación PsicAP. (2017). Impacto económico y carga de los trastornos mentales comunes en España: una revisión sistemática y crítica. Ansiedad y Estrés, 23(2-3), 118-123. https://doi.org/10.1 016/j.anyes.2017.10.003

Salari, N., Hosseinian-Far, A., Jalali, R., Vaisi-Raygani, A., Rasoulpoor, S., Mohammadi, M., Rasoulpoor, S., \& Khaledi-Paveh, B. (2020). Prevalence of Stress, Anxiety, Depression Among the General Population During the COVID-19 Pandemic: A Systematic Review and MetaAnalysis. Globalization and Health, 16(57). https:// doi.org/10.1186/s12992-020-00589-w

Sandín, B., Chorot, P., \& Valiente, R. M. (2012). Transdiagnóstico: nueva frontera en Psicología Clínica. Revista de Psicopatología y Psicología Clínica, 17(3), 185-203. http://e-spacio.uned.es/fez/eserv/bibliuned: Psicopat-2012-17-3-6005/Documento.pdf

Sanz, J., \& Navarro, M. E. (2003). Propiedades psicométricas de una versión española del Inventario de Ansiedad de Beck (BAI) en estudiantes universitarios. Ansiedad y Estrés, 9(1), 59-84. https://psycnet.apa.org/record/200399798-006

Sanz, J., Paz, M., \& Fortún, M. (2012). El Inventario de ansiedad de Beck (BAI): propiedades psicométricas de la versión española en pacientes con trastornos psicológicos. Psicología Conductual, 20(3), 563-583. https://www.behavioralpsycho.com/wp-content/ uploads/2019/08/05.Sanz_20-3oa-1.pdf

Stagnaro, J. C., Cía, A., Vázquez, N., Vommaro, H., Nemirovsky, M., Serfaty, E., Ezequiel, S., Medina, M. E., Benjet, C., Aguilar-Gaxiola, S., \& Kessler, S. (2018). Estudio epidemiológico de salud mental en población general de la República Argentina. Vertex. Revista Argentina de Psiquiatría, 29(142), 275-299. http:// www.editorialpolemos.com.ar/docs/vertex/vertex142. pdf\#page $=36$

Steer, R. A., Clark, D. A., Beck, A. T., \& Ranieri, W. F. (1995). Common and Specific Dimensions of Self-Reported Anxiety and Depression: A Replication. Journal of Abnormal Psychology, 104(3), 542-545. https://doi.org/ 10.1037/0021-843X.104.3.542

Steer, R. A., Clark, D. A., Beck, A. T., \& Ranieri, W. F. (1999). Common and Specific Dimensions of Self-Reported Anxiety and Depression: The BDI-II versus the BDIIA. Behaviour Research and Therapy, 37(2), 183-190. https://doi.org/10.1016/S0005-7967(98)00087-4

Tabachnick, B. G., \& Fidell, L. S. (2001). Using Multivariate Statistics. Allyn and Bacon.

Trevethan, R. (2017). Intraclass Correlation Coefficients: Clearing the Air, extending some Cautions, and Making some Requests. Health Services and Outcomes Research Methodology, 17(2), 127-143. https://doi.org/ 10.1007/s10742-016-0156-6

Vizioli, N. A., \& Pagano, A. E. (2020). Adaptación del Inventario de Ansiedad de Beck en población de Buenos Aires. Interacciones. Revista de Avances en Psicología, 6(3), e171. https://doi.org/10.24016/2020.v6n3.171

Voorhees, C. M., Brady, M. K., Calantone, R., \& Ramirez, E. (2016). Discriminant Validity Testing in Marketing: An Analysis, Causes for Concern, and Proposed Remedies. Journal of the Academy of Marketing Science, 44(1), 119-134. https://doi.org/10.1007/s11747-015-0455-4

World Medical Association. (2013). Declaration of Helsinki. Ethical Principles for Medical Research Involving Human Subjects. JAMA Network, 310(20), 2191-2194. https://doi.org/10.1001/jama.2013.281053 
Xiong, J., Lipsitz, O., Nasri, F., Lui, L., Gill, H., Phan, L., Chen-Li, D., Iacobucci, M., Ho, R., Majeed, A., \& McIntyre, R. S. (2020). Impact of COVID-19 Pandemic on Mental Health in the General Population: A Systematic Review. Journal of Affective Disorders, 277, 55-64. https://doi.org/10.1016/j.jad.2020.08.001
Zwick, W. R., \& Velicer, W. F. (1986). Comparison of Five Rules for Determining the Number of Components to Retain. Psychological Bulletin, 99(3), 432-442. https:/ /pascal-francis.inist.fr/vibad/index.php?action=get RecordDetail\&idt=8101602

\section{Alejandro Emilio Pagano}

Cátedra 059, Facultad de Psicología, Universidad de Buenos Aires, Argentina.

Licenciado en Psicología, especialista en Psicología Aplicada al Deporte, ayudante de primera de Teoría y Técnica de Exploración y Diagnóstico Psicológico Módulo I. Cátedra I: técnicas psicométricas (UBA). Investigador UBACyT.

ORCID: https://orcid.org/0000-0003-4817-9145

Autor corresponsal: paganoalejandro@gmail.com

Nicolás Alejandro Vizioli

Cátedra 059, Facultad de Psicología, Universidad de Buenos Aires, Argentina.

Licenciado en Psicología, ayudante de primera de Teoría y Técnica de Exploración y Diagnóstico Psicológico Módulo I. Cátedra I: técnicas psicométricas (UBA). Investigador UBACyT.

ORCID: https://orcid.org/0000-0002-6113-6847

nicovizioli@live.com 